\title{
ÓLEO DE ARROZ (ORYZA SATIVA): DEGOMAGEM E NEUTRALIZAÇÃO
}

\author{
J. I. ENGELMANN ${ }^{1}$, V. T. CREXI ${ }^{2}$ e M.M. MORAIS ${ }^{3}$ \\ ${ }^{1}$ Universidade Federal do Pampa, graduanda em Engenharia de Alimentos \\ ${ }^{2}$ Universidade Federal do Pampa, Prof ${ }^{\mathrm{a}}$. Dr ${ }^{\mathrm{a}}$ do curso de Engenharia de Alimentos \\ ${ }^{3}$ Universidade Federal do Pampa. Prof. Dr. do curso de Engenharia Química \\ E-mail para contato: jeny_ge@ hotmail.com
}

\begin{abstract}
RESUMO - O óleo de arroz é obtido a partir do farelo de arroz, tendo como diferencial em relação aos demais óleos a presença de $\gamma$-orizanol. Para que o óleo de arroz esteja apto ao consumo é necessário que este passe pelo processo de refino. entre as etapas do refino tem-se a degomagem e a neutralização.. A degomagem visa a separação dos fosfolipídeos presentes no óleo de arroz, obtendo-se como subproduto a lecitina. A neutralização visa reduzir a acidez livre presente no óleo. Com base no exposto, este trabalho teve como objetivo estudar as etapas de degomagem e neutralização do óleo de arroz, a fim de obter um óleo com menor acidez livre e teor de fósforo e maior teor de $\gamma$-orizanol. Os experimentos foram realizados a partir de um planejamento experimental fatorial. As variáveis de estudo para a degomagem foram a temperatura $\left(60\right.$ e $\left.80^{\circ} \mathrm{C}\right)$ e o percentual de água (1 e 5\%). Para a neutralização as variáveis de estudo foram temperatura $\left(60\right.$ e $\left.80^{\circ} \mathrm{C}\right)$ e percentual de excesso de $\mathrm{NaOH}(20$ e $40 \%)$. No estudo da degomagem pode-se verificar que nas condições de $5 \%$ de água e $60^{\circ} \mathrm{C}$ obteve-se um óleo com menor teor de fósforo, $2,4 \mathrm{mg} / 100 \mathrm{~g}$. No estudo da etapa de neutralização foi possível observar que a melhor região de trabalho situa-se próxima à condição de $40 \%$ de excesso de

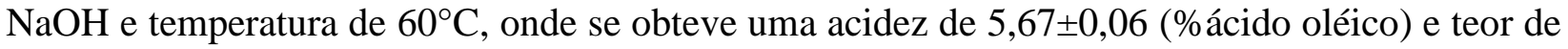
$\gamma$-orizanol de 1,35 $\pm 0,09$.
\end{abstract}

\section{INTRODUÇÃO}

O arroz é um dos mais importantes cereais produzidos no mundo, principalmente na Ásia e na América Latina, onde constitui a base da alimentação da maior parte da população (NAVARRO et al., 2007).

O óleo de arroz (Oryza sativa) é o produto obtido a partir do farelo de arroz, cujo teor de óleo pode variar entre 12 e 18\%, dependendo do cultivar e dos processos de extração (PAUCHARMENACHO et. al., 2007). O óleo do farelo de arroz tem sido considerado superior por suas características químicas, sendo equiparável aos óleos de soja, milho e algodão. Sendo rico em vitaminas, apresenta baixa incidência de ingredientes responsáveis pelo colesterol, além de alto 
teor de tocoferois (vitamina E) que garantem alta estabilidade, retardando a rancidez e o aparecimento de sabores indesejáveis. (RODRIGUES,2004).

O óleo de arroz apresenta um diferencial em relação aos demais óleos devido a presença de um composto denominado de $\gamma$ - orizanol, ao qual tem sido atribuídos efeitos antioxidantes hipocolesterolêmico. O conteúdo de $\gamma$-orizanol no óleo de farelo de arroz bruto varia entre 1-2\%, mas durante a etapa de neutralização do refino químico, este composto fica retido na borra (PAUCHAR-MENACHO et. al., 2007).

Para que se obtenha um óleo próprio para o consumo, é necessário que este passe pelas etapas de refino, sendo elas, degomagem, neutralização, branqueamento, winterização e desodorização (CREXI, 2009).

A degomagem tem como finalidade remover os fosfatídios presentes no óleo de arroz, além de facilitar o armazenamento e transporte do óleo cru, produz fosfatídios como subproduto valioso, sendo conhecido comercialmente como lecitina, utilizada como emulsificante. Esta etapa facilita ainda, o refino alcalino, aumentando o rendimento e a qualidade do óleo final, diminui a poluição por água ácida depois do desdobramento da borra (CALHEIROS, 2007).

A degomagem pode ser realizada fazendo-se uso de ácido, água, ou união destes, sendo que a degomagem com água corresponde à forma mais simples de promover a redução dos fosfatídeos, principalmente quando deseja-se extrair a lecitina, eliminando-se a necessidade de purificação desta devido à não utilização de ácidos no processo de degomagem (CASTEJON, 2010).

A neutralização do óleo de arroz consiste em fazer reagirem os ácidos graxos livres, responsáveis pela acidez do óleo, com uma solução de hidróxido de sódio. Estes ácidos graxos são então transformados em sabões, que serão removidos do óleo neutro por processo físico (centrifugação). Neste processo consegue-se também uma remoção de fosfatídeos não hidratáveis (DORSA, 2004). Os ácidos graxos livres, quando presentes em concentrações elevadas no óleo, possibilitam a ocorrência de uma série de reações, dentre as quais pode-se citar a formação de sabores e odores desagradáveis. Logo, pode-se dizer que acidez do óleo indica a qualidade do mesmo (PESTANA, et al. 2008).

Com base no exposto, este trabalho teve como objetivo realizar um estudo da etapa de degomagem e de neutralização do óleo de arroz, com o intuito de obter um óleo com menor acidez livre e teor de fósforo e maior teor de y-orizanol.

\section{METODOLOGIA}

A matéria - prima utilizada na realização dos experimentos foi o óleo bruto de arroz (Oryza sativa) e óleo degomado, as quais foram obtidas em uma indústria de óleos localizada no município de Camaquã-RS. 


\subsection{Metodologia estatística}

O estudo da etapa de degomagem e de neutralização foram realizados através de um planejamento de experimentos, sendo ordenados segundo uma Matriz de Planejamento Experimental Fatorial Completo $\left(2^{\mathrm{k}}\right)$, onde foram indicadas as variáveis de estudo com seus respectivos níveis, conforme apresentado por BOX et. al., (1978).

Os valores dos níveis dos fatores de estudos utilizados nas operações de degomagem e neutralização foram determinados através de testes preliminares, bem como dados encontrados na literatura. No estudo da etapa de degomagem foram determinadas como variáveis de estudo a temperatura e o percentual de água adicionado. As variáveis de resposta do planejamento experimental foram o teor de fósforo e a acidez livre em ácido oléico. Na Tabela 1 é apresentada a Matriz de Planejamento Experimental Fatorial Completo para a etapa de degomagem, com os valores das variáveis na forma codificada (cod.) e normal.

Tabela 1 - Matriz de planejamento experimental - degomagem

\begin{tabular}{ccccc}
\hline Experimentos & $\begin{array}{c}\text { Percentual de água } \\
\left(\% \mathrm{H}_{2} \mathrm{O}\right) \\
(\mathrm{cod})\end{array}$ & $\begin{array}{c}\text { Temperatura } \\
(\text { cod. })\end{array}$ & $\begin{array}{c}\text { Percentual de } \\
\text { água } \\
(\% \mathrm{p} / \mathrm{p})\end{array}$ & $\begin{array}{c}\text { Temperatura } \\
\left({ }^{\circ} \mathrm{C}\right)\end{array}$ \\
\hline 1 & -1 & -1 & 2 & 60 \\
2 & -1 & +1 & 2 & 80 \\
3 & +1 & -1 & 5 & 60 \\
4 & +1 & +1 & 5 & 80 \\
\hline
\end{tabular}

Fonte: Autor, 2014

Para a neutralização foram estudadas as variáveis temperatura e percentual de excesso de $\mathrm{NaOH}$ adicionado. Cabe ressaltar que este excesso, como o nome já diz, é um percentual adicionado além da quantidade estequiométrica necessária para neutralizar os ácidos graxos livres.

Na Tabela 2 é apresentada a Matriz de Planejamento Experimental Fatorial Completo para a etapa de neutralização, com os valores das variáveis na forma codificada (cod.) e normal. As variáveis resposta do planejamento experimental foram a acidez livre em ácido oléico e o teor de $\gamma$-orizanol. 
Tabela 2 - Matriz de planejamento experimental - neutralização

\begin{tabular}{ccccc}
\hline Experimentos & $\begin{array}{c}\text { Percentual de } \\
\text { exceso }(\% \mathrm{NaOH}) \\
(\text { cod.) }\end{array}$ & $\begin{array}{c}\text { Temperatura } \\
(\text { cod. })\end{array}$ & $\begin{array}{c}\text { Percentual de } \\
\text { excesso } \\
(\% \mathrm{NaOH})\end{array}$ & $\begin{array}{c}\text { Temperatura } \\
\left({ }^{\circ} \mathrm{C}\right)\end{array}$ \\
\hline 1 & -1 & -1 & $(20 \%)$ & $\left(60^{\circ} \mathrm{C}\right)$ \\
2 & -1 & +1 & $(20 \%)$ & $\left(80^{\circ} \mathrm{C}\right)$ \\
3 & +1 & -1 & $(40 \%)$ & $\left(60^{\circ} \mathrm{C}\right)$ \\
4 & +1 & +1 & $(40 \%)$ & $\left(80^{\circ} \mathrm{C}\right)$ \\
\hline
\end{tabular}

Fonte: Autor, 2014

\subsection{Procedimento experimental}

Para a realização da etapa de degomagem foram utilizadas amostras de óleo bruto de arroz, estas foram submetidas à diferentes temperaturas de aquecimento $\left(60^{\circ} \mathrm{C} \mathrm{e} 80^{\circ} \mathrm{C}\right)$, de acordo com cada experimento. À medida em que as amostras alcançavam tais temperaturas adicionava-se diferentes alíquotas de água, nas quantidades de 1 e $5 \%$ em relação a massa de óleo utilizada com constante agitação sob vácuo de $500 \mathrm{mmHg}$ durante 30 minutos. As amostras de óleo foram então centrifugadas a 3.000 rpm por 20 minutos para separação dos fosfolipideos.

Para a realização da etapa de neutralização, foram utilizadas amostras do óleo degomado, este, foi submetido a diferentes temperaturas de processo, $\left(60^{\circ} \mathrm{C}\right.$ e $\left.80^{\circ} \mathrm{C}\right)$. A quantidade de solução de $\mathrm{NaOH} \mathrm{18 \%} \mathrm{(massa/volume)} \mathrm{foi} \mathrm{calculada} \mathrm{a} \mathrm{partir} \mathrm{da} \mathrm{quantidade} \mathrm{estequiométrica} \mathrm{de} \mathrm{NaOH}$ necessária para neutralizar os ácidos graxos livres, sendo adicionado ainda, uma certa quantidade em excesso de $\mathrm{NaOH}$ de acordo com o planejamento experimental, sendo que esta solução foi adicionada ao óleo aquecido, e a mistura permaneceu em contato durante $20 \mathrm{~min}$ sob vácuo de $500 \mathrm{mmHg}$ e constante agitação (500rpm). Por fim, o óleo foi centrifugado a $3000 \mathrm{rpm}$ e lavado três vezes com água a $95^{\circ} \mathrm{C}$.

\subsection{Metodologia analítica}

Os óleos bruto e degomado foram analisados quanto a acidez livre em ácido oléico (\%) [Ca $5^{\mathrm{a}}$-40], teor de fósforo [CF, Ca 12-55], foram determinados de acordo com os métodos da AOCS- American Oil Chemists's Society), 1997. O óleo neutralizado foi analisado em relação à acidez em ácido oléico [Ca $5^{\mathrm{a}}$-40] e teor de $\gamma$-orizanol [ método Bucci et al. 2003].

\section{RESULTADOS E DISCUSSÕES}

\subsection{Degomagem}

A amostra de óleo bruto apresentou teor de fósforo de 42,2 $\pm 3,6 \mathrm{mg} / 100 \mathrm{~g}$ e acidez livre em

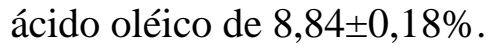


O teor de fósforo no óleo bruto apresentou valores cerca de duas vezes maior em relação ao padrão estabelecido para o óleo degomado $(20 \mathrm{mg} / 100 \mathrm{~g})$, evidenciando então, a necessidade deste óleo ser demogado.

Na Tabela 3 pode ser observada a matriz do planejamento experimental com os resultados obtidos a partir da realização de cada experimento.

Tabela 3 - Resultados obtidos através dos experimentos

\begin{tabular}{ccccc}
\hline Experimento & $\begin{array}{c}\text { Percentual de água } \\
(\% \mathrm{p} / \mathrm{p})\end{array}$ & $\begin{array}{c}\text { Temperatura } \\
\left({ }^{\circ} \mathrm{C}\right)\end{array}$ & $\begin{array}{c}\text { Teor de } \\
\text { fósforo }(\mathrm{mg} / 100 \mathrm{~g})\end{array}$ & $\begin{array}{c}\text { Acidez livre } \\
(\%)\end{array}$ \\
\hline 1 & 2 & 60 & $18,7 \pm 4,8$ & $9,0 \pm 1,0$ \\
2 & 2 & 80 & $39,4 \pm 1,3$ & $8,8 \pm 0,2$ \\
3 & 5 & 60 & $2,4 \pm 0,2$ & $9,0 \pm 0,4$ \\
4 & 5 & 80 & $32,1 \pm 3,3$ & $8,5 \pm 0,1$ \\
\hline
\end{tabular}

Fonte: Autor, 2014

Na Figura 1 é apresentado o gráfico de Pareto para o teor de fósforo, através do qual pode-se observar que as variáveis de estudo, temperatura e percentual de água, afetaram significativamente o teor de fósforo presente no óleo degomado em um nível de $95 \%(\mathrm{p}<0,05)$ de confiança. É possível afirmar ainda que a variável de estudo água afeta negativamente a resposta analisada, ou seja, causa redução no conteúdo de fósforo.

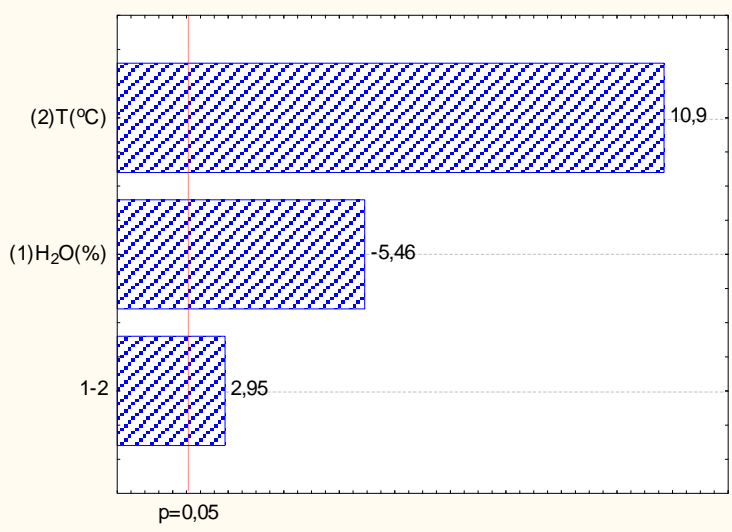

Figura 1 - Gráfico de Pareto - teor de fósforo

Na Figura 2 é apresentado o quadrado de resposta para a variável resposta teor de fósforo, a partir do qual as condições adequadas de trabalho são definidas. 


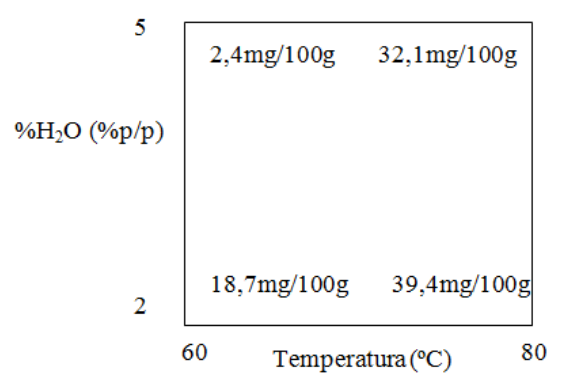

Figura 2 - Quadrado de resposta para o teor de fósforo

A partir da Figura 2 pode-se observar que produziu-se um óleo degomado com menor teor de fósforo $(2,4 \mathrm{mg} / 100 \mathrm{~g})$, utilizando-se $5 \%$ de água e temperatura de $60^{\circ} \mathrm{C}$.

\subsection{Neutralização}

Nesta etapa, utilizou-se como matéria-prima, o óleo degomado obtido a partir da melhor região de trabalho encontrada no estudo da etapa de degomagem, o qual apresentou acidez livre em ácido oléico de $9,0 \pm 0,4$ e teor de $\gamma$-orizanol de $1,65 \pm 0,04$. Na Tabela 4 pode ser observada a matriz de planejamento experimental com os respectivos resultados obtidos para cada experimento realizado.

Tabela 4-Resultados obtidos através dos experimentos

\begin{tabular}{ccccc}
\hline Experimento & $\begin{array}{c}\text { Percentual de } \\
\text { Excesso } \\
(\% \mathrm{NaOH})\end{array}$ & $\begin{array}{c}\text { Temperatura } \\
\left({ }^{\circ} \mathrm{C}\right)\end{array}$ & $\begin{array}{c}\text { Acidez livre } \\
(\%)\end{array}$ & $\begin{array}{c}\text { Teor de } \\
\gamma \text {-orizanol }(\%)\end{array}$ \\
\hline 1 & 20 & 60 & $5,94 \pm 0,19$ & $1,49 \pm 0,03$ \\
2 & 40 & 60 & $5,67 \pm 0,06$ & $1,35 \pm 0,09$ \\
3 & 20 & 80 & $6,03 \pm 0,11$ & $1,30 \pm 0,01$ \\
4 & 40 & 80 & $5,40 \pm 0,15$ & $0,98 \pm 0,02$ \\
\hline \multicolumn{4}{c}{ Fonte: Autor, 2014}
\end{tabular}

Na Figura 3 (a) e (b) são apresentados os gráficos de pareto para a acidez livre em ácido oléico e para o teor de $\gamma$-orizanol, respectivamente. No primeiro, pode ser observado que apenas a variável de estudo, excesso de $\mathrm{NaOH}$ influenciou significativamente a variável resposta, sendo que esta influencia é dita como negativa, ou seja, gerou redução da variável resposta (acidez livre). Já para a Figura (b) pode-se dizer que as variáveis de estudo, bem como a interação entre ambas, influenciaram negativamente a variável resposta, ou seja, reduziram o valor da variável resposta ( $\gamma$-orizanol). 


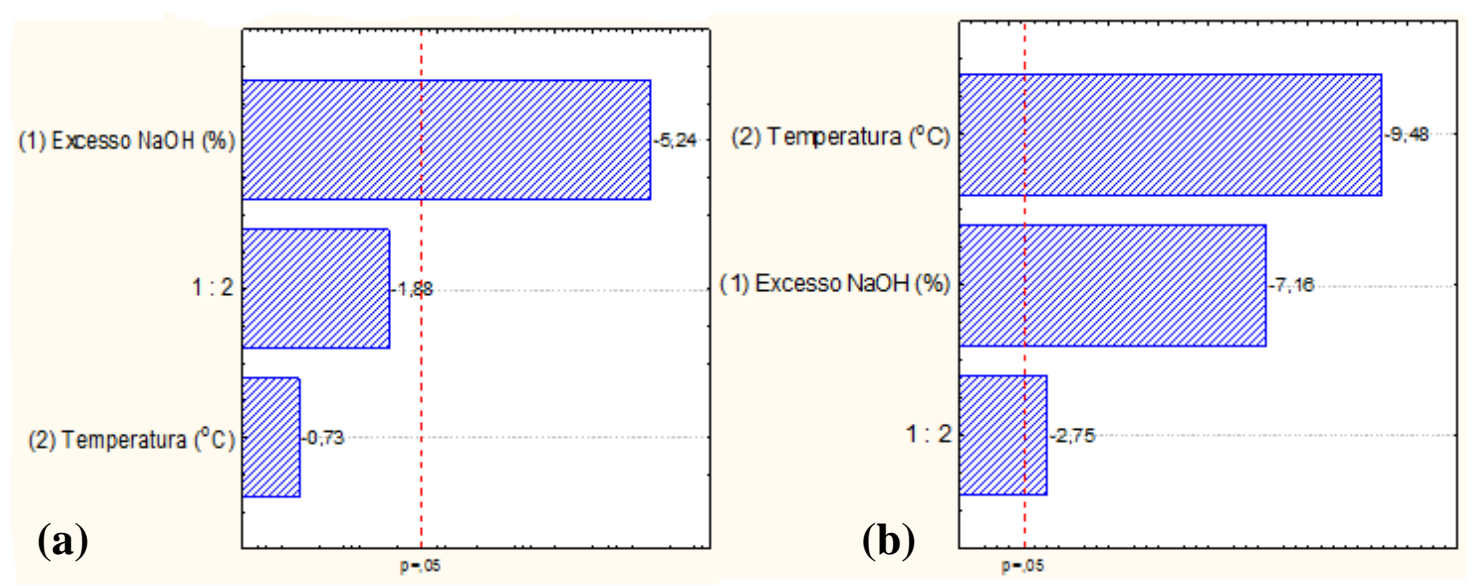

Figura 3 (a) e (b)- Gráfico de Pareto para as variáveis resposta acidez livre e teor de $\gamma$-orizanol, respectivamente

Nas Figuras 4 (a) e (b) podem ser observados os quadrados de resposta para as variáveis resposta acidez livre e teor de $\gamma$-orizanol, a partir dos quais as condições adequadas de trabalho são definidas.

(a)

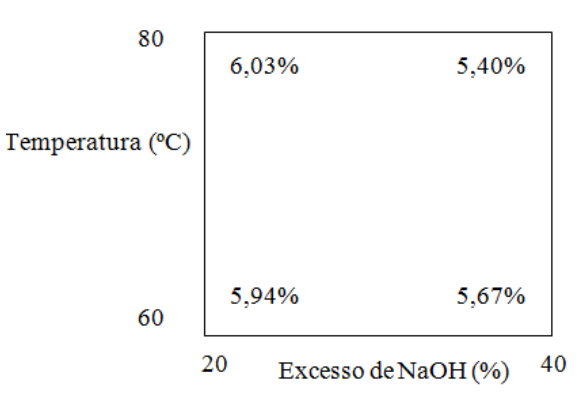

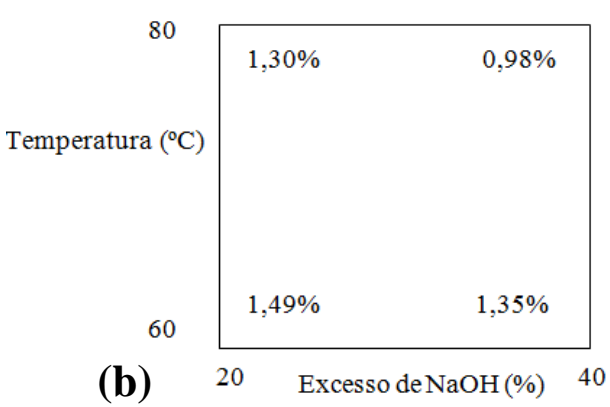

(b)

Figura 4 (a) e (b)- Quadrado de resposta para as variáveis acidez livre e teor de $\gamma$-orizanol, respectivamente

A partir da Figura 4 (a) pode-se observar que nas condições de $40 \%$ de excesso e temperatura de $80^{\circ} \mathrm{C}$ (Experimento 4) obteve-se uma maior redução da acidez livre do óleo, tendo-se como

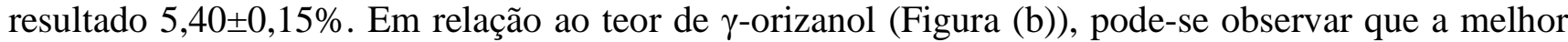
condição é a de $20 \%$ de excesso e $60^{\circ} \mathrm{C}$ (Experimento 1), no qual obteve menor redução, obtendo-se como resposta $(1,49 \pm 0,03 \%)$.

Pode-se observar ainda que nas condições $40 \%$ de excesso e $60^{\circ} \mathrm{C}$ (Experimento 2) obteve-se redução da acidez com valores próximos ao Experimento 4, com a vantagem de fazer uso de temperatura inferior à este, gerando menores gastos em termos de energia, gerando ainda, menores perdas do teor de $\gamma$-orizanol, sendo esta, a região de obtenção de melhores resultados. Cabe ressaltar ainda que os resultados obtidos para a acidez livre em ácido oléico não encontram-se dentro dos padrões exigidos pela ANVISA para este parâmetro, o qual é de no máximo $2 \%$. 


\section{9 a 22 de outubro de 2014 \\ Florianópolis/SC}

\section{CONSIDERAÇÕES FINAIS}

$\mathrm{Na}$ degomagem do óleo de arroz verificou-se que na temperatura de $60^{\circ} \mathrm{C}$ com $5 \%$ de adição de água (Experimento 1) obteve-se um óleo degomado com baixo teor de fósforo $(2,4 \mathrm{mg} / 100 \mathrm{~g})$.

$\mathrm{Na}$ etapa de neutralização verificou-se que na temperatura de $80^{\circ} \mathrm{C}$ e adição de $40 \%$ de excesso obteve-se uma maior redução da acidez livre do óleo de arroz $(5,40 \pm 0,15)$, porém, quando observa-se os resultados obtidos em relação ao teor final de $\gamma$-orizanol, tem-se como melhor condição o uso de temperatura de $60^{\circ} \mathrm{C}$ e adição de $20 \%$ de soda a qual confere menores perdas durante a neutralização $(1,49 \pm 0,03)$.Em relação à melhor condição para ambas variáveis resposta pode-se dizer

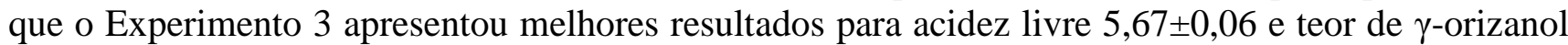
$1,35 \pm 0,09$, porém, deve ser observado que os valores obtidos para a variável acidez livre não encontram-se ainda, dentro dos padrões exigidos pela ANVISA. Pode-se concluir, que com maiores excessos de $\mathrm{NaOH}$ obtêm-se um óleo com menor acidez, porém com maior redução do teor de $\gamma$ orizanol. Logo, novos experimentos devem ser realizados ao redor destas condições para se buscar uma região ótima de trabalho.

\section{REFERÊNCIAS BIBLIOGRÁFICAS}

BUCCI, R., MAGRI, A.L, MARINI, F(2003). Comparison of three spectrophotometric methods for the determination of $\gamma$-oryzanol in rice bran oil. Analytical and Bioanalytical Chemistry. 375,p.1254-1259, 2003.

CALHEIROS, M.N.; Extração de orizanol da borra de neutralização do óleo de farelo de arroz. UFSC. Florianópolis - SC, 2007.

CASTEJON, L. V.; Estudo da clarificação da lecitina de soja. Dissertação de mestrado. Universidade de Uberlândia. Minas Gerais, 2010

CREXI, V. T., GRUNENNVALDT, F., SOARES, L., PINTO, L. A. A Deodorisation process variables for croaker (M. furnieri) oil. Food Chemistry, v. 114, p. 396-401,2009.

DORSA, R. Tecnologia de óleos vegetais. 1. ed. São Paulo: Ideal, 2004. 463p.

NAVARRO, F. M. S. et al. ; Desacidificação do óleo de farelo de arroz durante o processo de extração líquido-líquido. Estudo da variação de solventes. Exacta, vol. 5, núm. 1, janeiro-junho, 2007, pp. 163-167.

PAUCHAR-MENACHO, L.M, SILVA, L.H., SANT'ANA, A.S., GONÇALVES, L.A. Refino do óleo de farelo de arroz (Oryza Sativa L.) em condições brandas para preservação do $\gamma$ orizanol. Ciencia e Tecnologia de Alimentos. 27,45-53p, 2007.

RODRIGUES, C.E.C.; Desacidificação do oleo de farelo de arroz por extração liquido-liquido. Tese de Doutorado. Unicamp. Campinas-SP, 2004. 